\title{
An Investigation of the Structure of Amorphous Co-P Alloys. 2: EXAFS at the Phosphorous K-edge, a Feasibility Study
}

\author{
Mi. Nuding, P. Lamparter, S. Steeb, F. Neißendorfer ${ }^{\mathrm{a}}$, and F. Schäfers ${ }^{\mathrm{b}}$ \\ Max-Planck-Institut für Metallforschung, Seestr. 92, D-70174 Stuttgart \\ ${ }^{\text {a }}$ Institut für Physik der Universität Potsdam, Am Neuen Palais 10, D-14469 Potsdam \\ ${ }^{b}$ Berliner Elektronenspeicherring-Gesellschaft für Synchrotronstrahlung mbH (Bessy), \\ Lentzeallee 100, D-14195 Berlin
}

Z. Naturforsch. 53 a, 848-854 (1998); received September 12, 1998

\begin{abstract}
Amorphous $\mathrm{Co}_{100-x} \mathrm{P}_{x}$ foils in the concentration range $13.4 \leq x \leq 26$ were produced by electrodeposition. The extended X-ray absorption fine structure (EXAFS) at the phosphorous K-edge was measured in transmission mode. The phase shift was determined from the EXAFS spectrum of a crystalline $\mathrm{Co}_{2} \mathrm{P}$ reference sample. The EXAFS spectra of the amorphous Co-P alloys are characterized mainly by one single contribution from a shell of Co-atoms at a distance of $2.3 \AA$ around the P-atoms. A small additional contribution at a shorter distance of $2.0 \AA$ may be a hint on some direct P-P neighbours. The evaluation of reliable coordination numbers from the EXAFS spectra of the amorphous alloys is not possible.
\end{abstract}

\section{Introduction}

For many amorphous alloys the determination of all atomic distances and partial coordination numbers is difficult because there are not enough independent methods available for their structural characterization, e.g. due to the lack of stable isotopes for a contrast variation in neutron diffraction experiments. In such cases the combination of EXAFS with X-rayand neutron diffraction may provide a solution of this general problem. EXAFS is very promising, because it allows to select the absorption edge of a specific component in the alloy, and thus to probe the immediate surrounding of the absorbing atom [1]. It was the motivation for the present work to supplement the results from X-ray- and neutron diffraction experiments with amorphous Co-P alloys [2, 3] by EXAFS experiments at the phosphorous K-edge. Very few EXAFS studies at the absorption edge of the metalloid atoms in metal-metalloid glasses are known, most probably because the experiments are difficult to be performed. If one works in transmission mode, the rather soft Xrays require very thin samples with a thickness of the order of $1 \mu \mathrm{m}$. EXAFS at the P-K-edge of a sputtered $\mathrm{Co}_{80} \mathrm{P}_{20}$ sample has been reported in [4]. It is well known that for amorphous alloys the quantita-

Reprint requests to Dr. P. Lamparter; Fax: +497112095420. tive evaluation of atomic distances and (even more) of coordination numbers from EXAFS data is much more complicated than from diffraction data. In this respect the present investigation may be regarded as a feasibility study of the EXAFS method applied to the phosphorous K-edge. An extensive compilation of the relevant literature is presented.

\section{Theoretical Fundamentals}

The fine structure of the linear X-ray absorption coefficient within the energy range of several hundred $\mathrm{eV}$ above the absorption edge depends very sensitively on the immediate atomic surrounding of the absorbing atom [1]. It is treated theoretically by the so called short range order theory [5 - 13]. The linear absorption coefficient $\mu(E)$ in the energy range above the absorption edge is separated into an unmodulated share $\mu_{0}(E)$ and into a share $\chi(E)$ which describes the EXAFS oscillations.

$$
\mu(E)=\mu_{0}(E) \cdot[1+\chi(E)] .
$$

Usually, the oscillations are presented as a function of the modulus $k$ of the wave vector $\boldsymbol{k}$ of the photoelectron:

$$
k=\sqrt{\frac{2 m}{\hbar^{2}} \cdot\left(E-E_{0}\right)},
$$


where $m$ is the electron mass and $E_{0}$ is the energy for the production of a photoelectron with the wave vector $\boldsymbol{k}=0$. In the framework of the short range order theory the normalized EXAFS-modulation $\chi(k)$ can be written as

$$
\begin{aligned}
\chi(k)=\sum_{j} & \frac{N_{j} S_{i}(k)}{k R_{j}^{2}} F_{j}(k) \exp \left[-2 \sigma_{j}^{2} k^{2}\right] \\
& \times \exp \left[-2 R_{j} / \lambda_{j}(k)\right] \sin \left(2 k R_{j}+\phi_{i j}(k)\right)
\end{aligned}
$$

or

$$
\chi(k)=\sum_{j} A_{j}(k) \cdot \sin \left(2 k R_{j}+\phi_{i j}(k)\right) .
$$

The sum includes all coordination spheres $j$, each of them containing $N_{j}$ atoms of type $j$ at the distance $R_{j}$ from the absorbing atom of type $i$.

The amplitude reduction factor $S_{i}(k)$ takes into account that the photoelectron which is produced during the absorption of an X-ray photon can lose more or less energy to a valence electron of the absorbing atom by so called shake up/off processes [14-16]. The backscattering amplitude $F_{j}(k)$ of the backscattered electron wave determines very strongly the shape of the EXAFS-amplitude function and can be used to identify the type of the backscattering atoms [17]. The two exponential terms in (3) are attenuation factors. The first term mainly describes the damping of $\chi(k)$ with increasing $k$-values by means of a Debye-Waller factor, containing the damping parameter $\sigma_{j}$. It takes into account the thermal vibrations of the atoms and the static mean square deviation of a Gaussian distance distribution of the backscattering atoms [18 - 21]. The second attenuation term in (3) takes into account the finite mean free path $\lambda_{j}(k)$ of the photoelectrons. Inelastic scattering of the photoelectrons and the finite lifetime of the unoccupied hole within the absorbing atom mean reduced interference ability between outgoing and backscattered wave and cause an attenuation of $\chi(k)$ mainly at small $k$-values $[15,22,23]$. The total phase of the sine-function in (3) consists of a geometrical share $2 k R_{j}$ and a phase shift $\phi_{i j}(k)$ which is caused by interaction of the electron wave with the electron shells of the absorbing atom and the backscattering atom [24].

Fourier-transformation of $\chi(k)$ yields a radial density function $F(R)$ of the backscattering atoms around the absorbing atom [25 - 27].

$$
F(R)=\frac{1}{\sqrt{2 \pi}} \int_{k_{\min }}^{k_{\max }} W(k) k^{n} \chi(k) e^{2 i k R} \mathrm{~d} k .
$$

The window function $W(k)$ is applied for a smooth transition from zero to unity at the integration limits $k_{\min }$ and $k_{\max }$ in order to reduce the termination effect $[7,28,29]$. The weighting factor $k^{n}$, with $n=1,2$ or 3 , compensates for the strong attenuation of $\chi(k)$ at large $k$-values which is necessary mainly for backscattering atoms with small atomic number $[8,30]$.

The modulus $|F(R)|$ of the radial density function is a measure for the number density of the neighbouring atoms. The positions of the peaks of $|F(R)|$, however, occur at distances which are smaller than the true atomic distances, since during the Fourier-transformation the phase shift $\phi_{i j}(k)$ was neglected [31]. Therefore, the determination of atomic distances usually is done by fitting a theoretical model function to $\chi(k)$.

\section{Experimental}

\subsection{Sample Preparation}

Co-P samples were prepared with P-contents in the range 13.4 at $\%<c_{\mathrm{p}}<26$ at $\%$. For the EXAFS measurements at the P-K-edge very thin samples had to be produced. Using the electrodeposition method as described in $[2,3]$ it was possible to prepare Co-P foils with a thickness of only $1 \mu \mathrm{m}$. For the experimental determination of the phase shift two crystalline specimens were used. One was obtained by sputtering from an $\mathrm{Co}_{2} \mathrm{P}$-target onto a $6.2 \mu \mathrm{m}$ thick mylar foil and the other by crystallization of an amorphous $\mathrm{Co}_{75} \mathrm{P}_{25}$ foil $\left(500^{\circ} \mathrm{C} ; 24 \mathrm{~h}\right.$; atmosphere: Argon 5N).

\subsection{EXAFS Experiments}

The EXAFS experiments at the phosphorous $\mathrm{K}$ edge of the amorphous and crystalline Co-P alloys were done in transmission mode using the KMC equipment at BESSY (Berlin, Germany) [32 - 34]. It uses an In-Sb double crystal monochromator which works in UHV. The beam cross section at the specimen amounts to $2 \times 3 \mathrm{~mm}^{2}$. The primary intensity $I_{0}$ was measured by recording the photo current from a Cu-grid in front of the specimen. The transmitted intensity $I$ was measured either by a GaAsP/Au Schottky diode (type G2119, Hamamatsu Inc.) or by a 


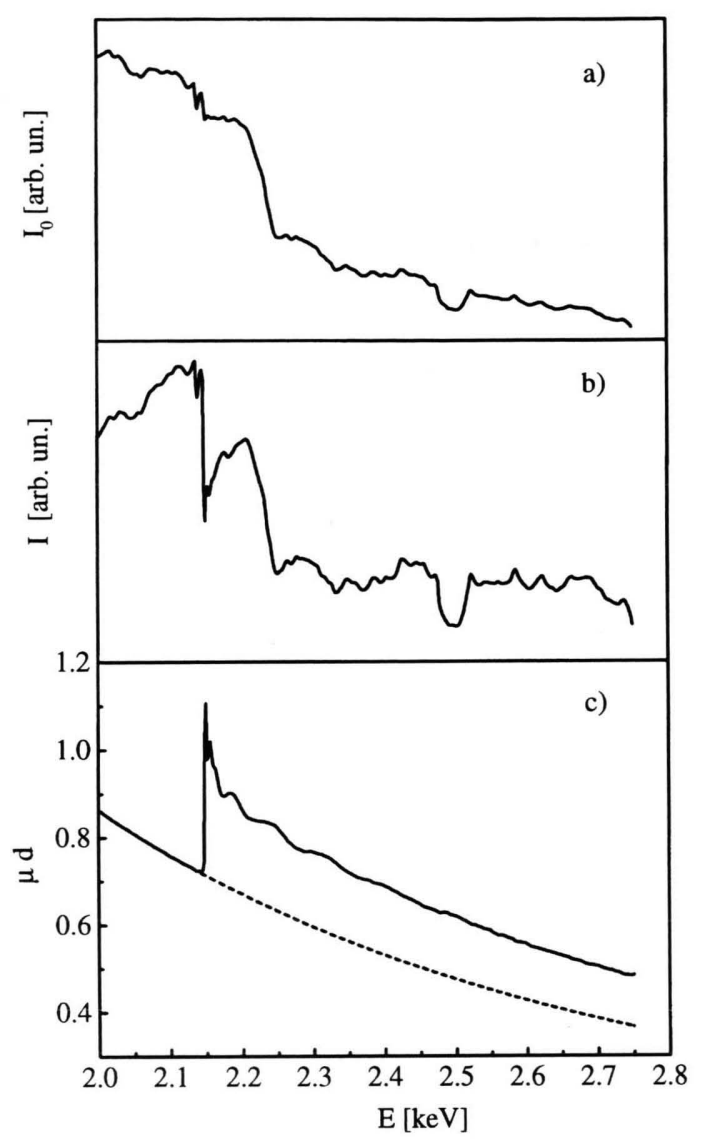

Fig. 1. Amorphous $\mathrm{Co}_{75} \mathrm{P}_{25}$ : EXAFS experiment. a) intensity of incident beam $I_{0}$, b) intensity of transmitted beam $I$, c) absorption $\mu d=\ln \left(I_{0} / I\right)$. - - - fit to the range in front of the $\mathrm{P}-\mathrm{K}$ edge.

silicon diode (type XUV 100C, United Detector Technologies) which both are very suitable for the phosphorous K-edge [35, 36]. The EXAFS spectra were measured between $2 \mathrm{keV}$ and $2.75 \mathrm{keV}$ in $1.5 \mathrm{eV}$ steps for one second each. From each alloy 50 spectra were measured and averaged. The adjustment of the KMC station was checked using the sulfur K-edge and the gold M-edge.

\subsection{Data Reduction}

Figure 1a shows the intensity $I_{0}$ which is determined by the spectral characteristics of the radiation source, the reflectivity of the monochromator, and by the other optical components of the beam line. Figure $1 \mathrm{~b}$ shows the intensity transmitted through an amorphous $\mathrm{Co}_{75} \mathrm{P}_{25}$ samle with the phosphorous $\mathrm{K}$-edge at $2.148 \mathrm{keV}$. Figure 1c finally shows

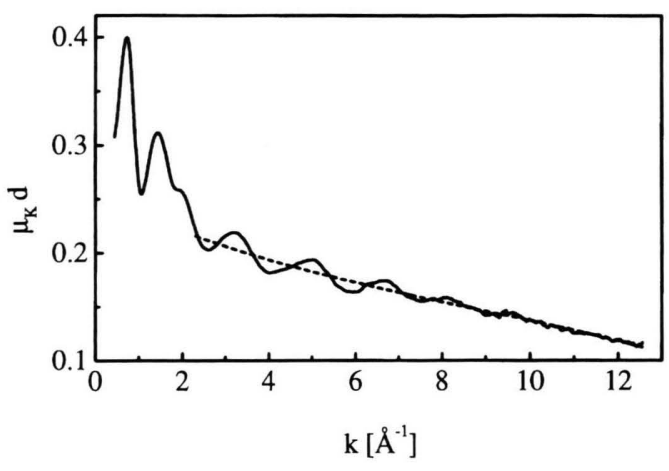

Fig. 2. Amorphous $\mathrm{Co}_{75} \mathrm{P}_{25}$ : phosphorous K-absorption. - - - polynomial fit, representing the unmodulated part.

$\mu d=\ln \left(I_{0} / I\right)$, where $d$ is the specimen thickness. Directly above the edge the rather strong oscillating XANES region occurs which is followed by the EXAFS region. The dashed line shows the contribution $\mu_{\mathrm{b}}(E)$ to the absorption, which is caused by the cobalt atoms and by the L- and M-absorption of the phosphorous atoms. It was determinded by fitting a Victoreen-function [37] to the measured $\mu d$ function in front of the P K-edge:

$$
\mu_{\mathrm{b}}(E)=a_{1} \cdot\left(\frac{h c}{E}\right)^{3}+a_{2} \cdot\left(\frac{h c}{E}\right)^{4},
$$

where $a_{1}$ and $a_{2}$ are the fitting parameters (see [38]).

Subtraction of the background $\mu_{\mathrm{b}}(E)$ yields the corrected absorption $\mu_{\mathrm{K}}(E) d$, as shown in Fig. 2 versus the wave vector $\mathbf{k}$. The threshold energy $E_{0}$ was determined from the energy at half of the height of the absorption edge [39-42]. For all alloys we used $E_{0}=$ $2.148 \mathrm{keV}$. The unmodulated part $\mu_{0}(E) d$ in (1) was obtained by fitting a polynomial of the order of three to the EXAFS region (dashed line in Figure 2). Finally, the EXAFS function $\chi(\mathrm{k})$ was calculated from (1) where the thickness $d$ cancels out.

\section{Results and Discussion}

Figure 3 shows as an example the $\chi(k)$ spectrum for amorphous $\mathrm{Co}_{75} \mathrm{P}_{25}$ together with the modulus $|F(R)|$ of the complex Fourier-transform. For twelve further P-concentrations the corresponding spectra are presented in [2]. The EXAFS oscillations can be well described by one single frequency which indicates that in amorphous Co-P alloys only one atomic distance around the $\mathrm{P}$-atoms is dominant. $|F(R)|$ shows 

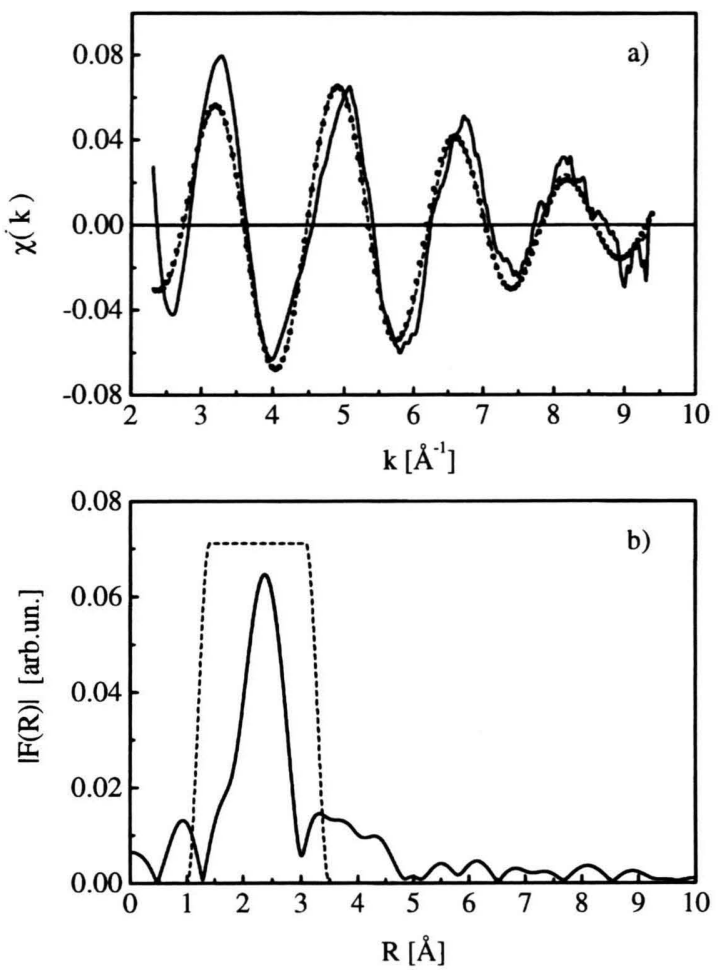

Fig. 3. Amorphous $\mathrm{Co}_{75} \mathrm{P}_{25}$ : a) normalized EXAFS-oscillations $\chi(k)$. - $\chi(k)$ experimental, - - - $\chi_{1}(k)$ back-transformed first shell, $\bullet$ fit to $\chi_{1}(k)$ according to (3). b) modulus of the Fourier transform of $\chi(k)$. - - - window function for the back-transformation of the first coordination shell.

a main peak at $R=2.25 \AA$ which reflects the distribution of nearest neighbour atoms around a central phosphorous atom. The second sphere in $|F(R)|$ is very weak and further spheres cannot be detected since the mean free path of a photoelectron only amounts to $10 \AA$ and therefore only a region with a radius of about $5 \AA$ around the absorbing atom can be detected $[10,15]$.

\subsection{Atomic Distances}

Due to the phase shift $\phi_{i j}(k)$ in (3), which is not taken into account in the Fourier-transformation (4), the peaks of $|F(R)|$ in Fig. 3b are shifted to smaller $R$ values. Therefore the distances were determined by a fitting procedure in $k$-space. The $|F(R)|$ function was back-transformed, where by the use of a Hanning-type window function $W(R)$ [2] (dashed curve in Fig. 3b) the contribution of the first coordination sphere was isolated [5, 30, 43, 44]. Furthermore, for short distances the fit within $k$-space yields better results than
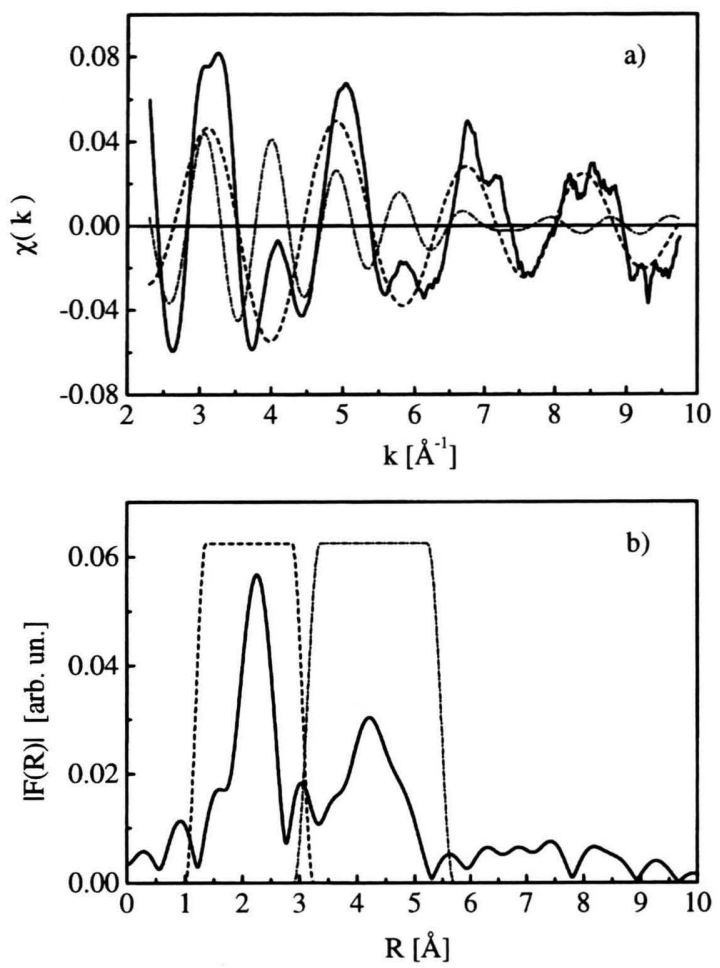

Fig. 4. Crystalline $\mathrm{Co}_{2} \mathrm{P}$ : a) normalized EXAFS-oscillations $\chi(k)$. - $\chi(k)$ experimental, - - - $\chi_{1}(k)$ back-transformed first shell, - - - $\chi_{2}(k)$ back-transformed second shell. b) modulus of the Fourier transform of $\chi(k)$. - - - window function for the first coordination shell, - . - window function for the second coordination shell.

in $R$-space [31, 43, 45]. By the back-transformation of the first sphere also multiple scattering effects are eliminated [46 - 51]. The back-transformed function $\chi_{1}(k)$ is shown in Fig. 3a as dashed line. For the fit to $\chi_{1}(k)$, according to (3), the phase shift $\phi_{i j}(k)$ was determined by employing the concept of the transferability of phase shifts.

An EXAFS spectrum of a reference substance with well known atomic distances has to be measured $[8,52,53]$. From this reference spectrum the phase shift $\phi_{i j}(k)$ can be determined by fitting (3) to the EXAFS oscillations $\chi(k)$ using the known atomic distances. The distances for the amorphous alloys then can be obtained by transferring the phase shift to the amorphous alloy and the subsequent fitting of the EXAFS equation to the back-transformed $\chi_{1}(k)$ function $[9,10,20,54,55]$. The transferability of phase shifts, as determined with crystalline reference substances, to amorphous alloys is well known and reliable $[31,43,52,56,57]$, presupposed that the sur- 
Table 1. Orthorhombic $\mathrm{Co}_{2} \mathrm{P}$ : atomic distances $R$ and coordination numbers $N$.

\begin{tabular}{lllllll}
\hline & \multicolumn{4}{c}{$\mathrm{CO}^{\mathrm{I}}$} & \multicolumn{3}{c}{ Neighbour } & \multicolumn{2}{c}{${ }^{\mathrm{Co}} \mathrm{II}$} \\
Central & $R[\AA]$ & $N$ & $R[\AA]$ & $N$ & $R[\AA]$ & $N$ \\
\hline $\mathrm{Co}^{\mathrm{I}}$ & 2.54 & 2 & 2.665 & 6 & & \\
$\mathrm{Co}^{\mathrm{II}}$ & & & 2.93 & 4 & & \\
$\mathrm{P}$ & 2.21 & 4 & 2.434 & 5 & 3.27 & 2 \\
\hline
\end{tabular}

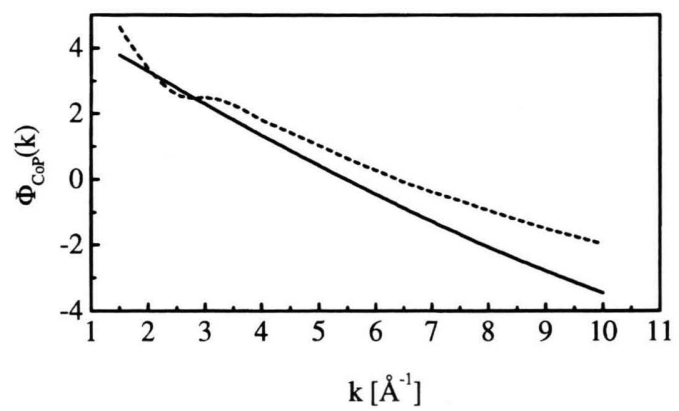

Fig. 5. Crystalline $\mathrm{Co}_{2} \mathrm{P}$ : Phase shift $\phi_{\mathrm{CoP}}(k)$. - experimental from crystalline $\mathrm{Co}_{2} \mathrm{P}$, - - - according to McKale.

roundings of the absorbing atoms in the reference substance and in the amorphous alloy are rather similar.

Figure 4a shows the EXAFS reference spectrum $\chi(k)$ for crystalline $\mathrm{Co}_{2} \mathrm{P}$, which contains compared to the amorphous alloys also contributions with higher frequencies from larger interatomic distances. The modulus $|F(R)|$ for crystalline $\mathrm{Co}_{2} \mathrm{P}$ in Fig. $4 \mathrm{~b}$ shows a second maximum at $R=4.21 \AA$ which was not observed with the amorphous alloy. The main peak at $R=2.25 \AA$ is narrower than in the amorphous case. For fitting of the back-transformed function $\chi_{1}(k)$ from the first sphere a linear fit routine was developed which uses the simplex method combined with the method of simulated annealing [2].

The atomic distances for crystalline $\mathrm{Co}_{2} \mathrm{P}$ are listed in Table $1[58,59]$. The $\mathrm{P}$-atoms are coordinated by 9 Co-neighbours, four $\mathrm{Co}^{\mathrm{I}}$-atoms at an average distance of $R_{\mathrm{Co} \text { P }}=2.21 \AA$ and five $\mathrm{Co}^{\mathrm{II}}$-atoms at an average distance of $R_{\mathrm{Co} \text { II }}=2.43 \AA$. The P-P distance is $3.27 \AA$ which means no direct P-P contact.

Figure 5 shows the $k$-dependence of the phase shift $\phi_{\mathrm{CoP}}$ as obtained with crystalline $\mathrm{Co}_{2} \mathrm{P}$ using the Co-P distances from Table 1. Figure 5 also presents the phase shift as tabulated by McKale et al. [60] which shows discrepancies compared to the experimental run especially at higher $k$-values. According to $[52,61,62]$ we prefer the experimental phase shift
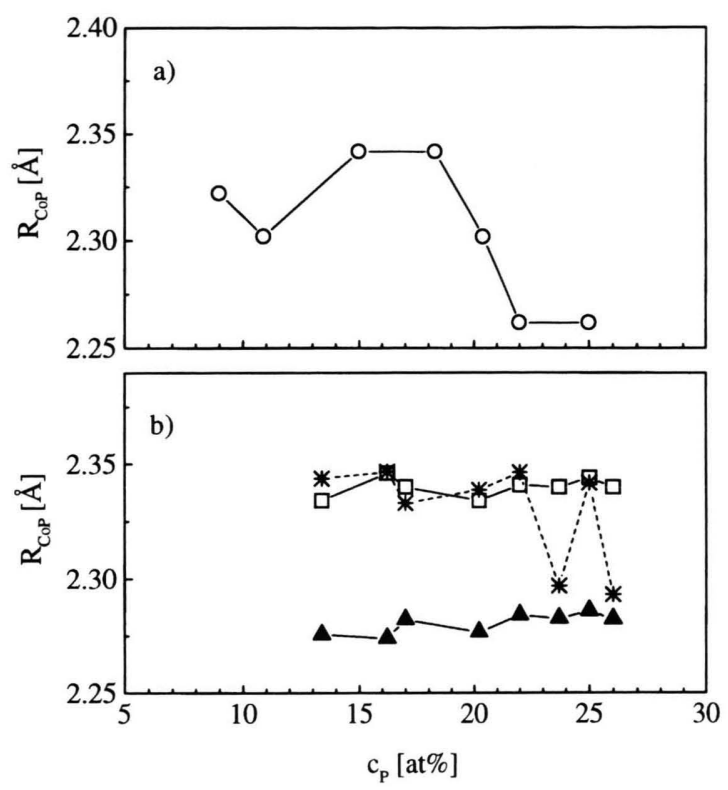

Fig. 6. Amorphous Co-P alloys: distances $R_{\mathrm{CoP}}$. a) from Xray and neutron diffraction $[2,3]$. b) from EXAFS. $\square$ from fit according to (3) with one Gaussian and experimental $\phi_{\mathrm{CoP}}(k), *$ with two Gaussians and experimental $\phi_{\mathrm{CoP}}(k)$,

$\boldsymbol{\Delta}$ with one Gaussian and McKale $\phi_{\mathrm{CoP}}(k)$. The lines in a) and b) are drawn as guide to the eye.

for the interpretation of the spectra obtained with the amorphous alloys.

A fit of the $\chi_{1}(k)$ function of the amorphous alloys with one single Gaussian contribution turned out to be not perfect [2]. However, using two Gaussians, namely a major contribution at $R_{1}=2.34 \AA$ and a small contribution at $R_{2}=2.01 \AA$, a perfect fit is possible as shown in Fig. 3a (dots). The main contribution at $R_{1}$ is in accordance with the Co-P distances of about $2.3 \AA$, which were obtained from diffraction $[2,3]$, whereas the distance $R_{2}$ is distinctly smaller. From the distances $R_{\mathrm{CoCo}}=2.58 \AA$ and $R_{\mathrm{CoP}}=2.26 \AA[2,3]$ an apparent diameter of the P-atoms can estimated as $R_{\mathrm{PP}}=1.94 \AA$, which is close to $R_{2}=2.01 \AA$. Thus one cannot exclude that in amorphous $\mathrm{Co}_{74} \mathrm{P}_{25}$ a small number of P-P pairs in close contact may exist.

Figure 6 shows for all amorphous alloys under investigation the obtained Co-P distances, in the upper part from diffraction $[2,3]$ and in the lower part from EXAFS. Above the eutectic concentration (19.9 at $\%$ P) the Co-P distances from diffraction become smaller, whereas the data from EXAFS do not depend significantly on the P-content. The Co-P distances as determined by diffraction fall into the region between 


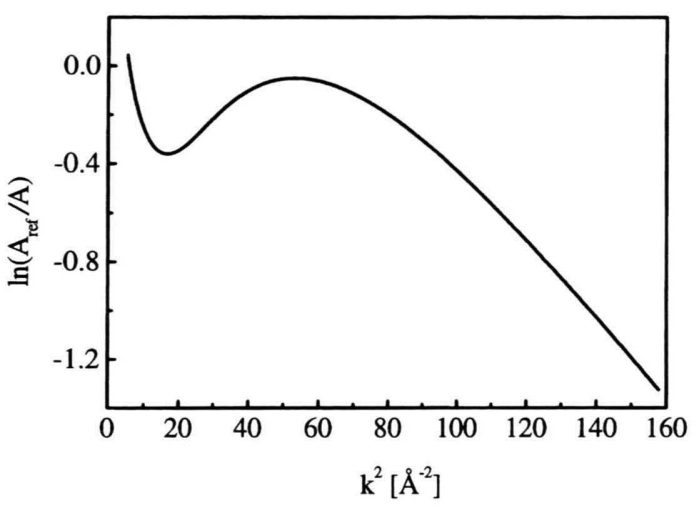

Fig. 7. Logarithmic plot of the ratio of the EXAFS amplitudes $A(k)$ (c.f. (3b)) of crystalline $\mathrm{Co}_{2} \mathrm{P}$ and amorphous $\mathrm{Co}_{75} \mathrm{P}_{25}$ versus $k^{2}$.

the EXAFS data obtained once with the phase shift from crystalline $\mathrm{Co}_{2} \mathrm{P}$ ( $\square$ in Fig. 6b) and once with the phase shift tabulated by Mc Kale [60] ( $\Delta$ in Fig. 6b). We state that the Co-P distances of the amorphous alloys fall in between the distances $R_{\mathrm{Co} \text { P }}$ and $R_{\mathrm{Col}}$.

\subsection{Partial Coordination Numbers}

It is well known that the determination of reliable coordination numbers of amorphous alloys from EXAFS hardly is possible, even if a crystalline reference standard is employed. This can be illustrated by means of the so called ratio method [63 - 67]. From (3) follows for the ratio of the amplitude functions $A_{\text {ref }}(k)$ and $A(k)$ of a crystalline reference substance and the amorphous alloy, respectively:

$\ln \left(\frac{A_{\mathrm{ref}}(k)}{A(k)}\right)=\ln \left(\frac{N_{\mathrm{ref}} \cdot R^{2}}{N \cdot R_{\mathrm{ref}}^{2}}\right)-2\left(\sigma_{\mathrm{ref}}^{2}-\sigma^{2}\right) k^{2},(6)$

[1] E. A. Stern, Phys. Rev. B10, 3027 (1974).

[2] Mi. Nuding, thesis work, University of Stuttgart 1993.

[3] Mi. Nuding, P. Lamparter, and S. Steeb, Z. Naturforsch. 53a, 841 (1998).

[4] A. M. Flank, P. Lagarde, D. Raoux, J. Rivory, and A. Sadoc, Proc. 4th Int. Conf. on Rapidly Quenched Metals, RQM4, Sendai 1981, the Japan Institute of Metals, Sendai 1982, p. 393.

[5] D. E. Sayers, E. A. Stern, and F. W. Lytle, Phys. Rev. Lett. 27, 1204 (1971).

[6] D. E. Sayers, F. W. Lytle, and E. A. Stern, in: Advances in X-ray Analysis, Plenum Press, New York 1970, p. 248.

[7] J. Wong, Mat. Sci. and Eng. 80, 107 (1986). where only the first coordination sphere is considered and where the mean free paths $\lambda(k)$ are assumed to be equal.

Figure 7 shows the corresponding plot of the ratio versus $k^{2}$ as obtained from crystalline $\mathrm{Co}_{2} \mathrm{P}$ and amorphous $\mathrm{Co}_{75} \mathrm{P}_{25}$. From the fact that this plot does not yield a straight line we have to conclude that (6) cannot be applied successfully. That means that the determination of partial coordination numbers from the EXAFS data is not possible in the present case.

\section{Conclusions}

A feasibility study of EXAFS spectroscopy at the phosphorous K-edge of amorphous electrodeposited $\mathrm{Co}_{100-x} \mathrm{P}_{x}$ alloys (13.4 $\leq x \leq 26$ ) was performed. It showed that, on the one hand, EXAFS oscillations with high quality can be obtained experimentally, but that, on the other hand, the difficulties in the extraction of structural parameters are connected with the data reduction procedures. Co-P distances were obtained by fitting the theoretical EXAFS equation to the normalized EXAFS spectrum in $k$-space using two Gaussians. The Co-P distances which were determined by means of EXAFS and by means of the diffraction methods were in good agreement. It was not possible to deduce reliable coordination numbers from the EXAFS spectra.

\section{Acknowledgements}

Thanks are due to BESSY, Berlin for the allocation of beam time. This work was supported by the Deutsche Forschungsgemeinschaft through grant La 686/1.

[8] E. A. Stern, D. E. Sayers, and F. W. Lytle, Phys. Rev. B11, 4836 (1975).

[9] C. A. Ashley and S. Doniach, Phys. Rev. B11, 1279 (1975).

[10] P. A. Lee and J. B. Pendry, Phys. Rev. B11, 2795 (1975).

[11] B. K. Teo, in: EXAFS: Basic Principles and Data Analysis, C. K. Jørgensen, M. F. Lappert, S. J. Lippard, J. L. Margrave, K. Nidenzu, H. Nöth, R.W. Parry, and H. Y. Yamatera, edts., Springer Verlag, Berlin 1996.

[12] R. Manaila and D. Macovei, J. Non-Cryst. Solids. 90, 383 (1987). 
[13] D. E. Sayers, in: Amorphous and Liquid Semiconductors, W. E. Spear, edt., G. G. Stevenson Ltd., Dundee 1977 , p. 61.

[14] E. A. Stern, S. M. Heald, and B. Bunker, Phys. Rev. Lett. 42, 1372 (1979).

[15] E. A. Stern, S. M. Heald, and B. A. Bunker, Phys. Rev. B21, 5521 (1980).

[16] K. D. Bomben, M. K. Bahl, J. K. Gimzewski, S. A. Chambers, and T. D. Thomas, Phys. Rev. A20, 2405 (1979).

[17] R. F. Pettifer and P. W. McMillan, Phil. Mag. 35, 871 (1977).

[18] R. B. Greegor and F. W. Lytle Phys. Rev. B20, 4902 (1979).

[19] P. Rabe, G. Tolkiehn, and A. Werner, J. Phys. C12, 899 (1979).

[20] P. Rabe, G. Tolkiehn, and A. Werner J. Phys. C12, 1173 (1979).

[21] P. Rabe, G. Tolkiehn, and A. Werner, Nucl. Instrum. Methods 171, 329 (1980).

[22] P. Eisenberger and B. Lengeler Phys. Rev. B22, 3551 (1980).

[23] B. K. Teo, J. Amer. Ceram. Soc. 103, 3990 (1981)

[24] B. Lengeler, Ber. Bunsenges. Phys. Chem. 90 (1986) 649.

[25] S. J. Gurman, J. Non-Cryst. Solids 17, 1541 (1982).

[26] J. W. Wong, F. W. Lytle, R. B. Greegor, H. H. Liebermann, J. L. Walter, and F. E. Luborsky, in: Rapidly Quenched Metals III, The Metals Society, London 1978 , p. 345.

[27] E. A. Stern, Y. Ma, O.H. Petitpierre, and C.E. Bouldin, Phys. Rev. B46, 687 (1992).

[28] C. Bingham, M. D. Godfrey, and J. W. Turkey, IEEE Trans. Audio and Electroacoust. 15, 58 (1967).

[29] K. Okasaka, H. Nasu, and K. Kamiya, J. Non-Cryst. Solids 136, 103 (1991).

[30] F. W. Lytle, D. E. Sayers, and E. A. Stern, Phys. Rev. B11, 4825 (1975).

[31] P. A. Lee, P. H. Citrin, P. Eisenberger, and B. M. Kincaid, Rev. Mod. Phys. 53, 769 (1981).

[32] J. Feldhaus, F. Schäfers, and W. Peatman, BESSY Jahrbuch 1986, p. 314.

[33] J. Feldhaus, F. Schäfers, and W. Peatman, SPIE Soft X-ray Optics and Technology 733, 242 (1986).

[34] J. Feldhaus, BESSY Technischer Bericht TB44, 1983.

[35] C. Storb, U. Dedek, W. Weber, B. Lengeler, and M. Schuster, Nuclear Instruments and Methods in Physics Research A306, 544 (1991).

[36] M. Krumrey and E. Tegeler, Rev. Sci. Instrum. 63, 797 (1992).

[37] J. A. Victoreen, J. Appl. Phys. 14, 95 (1943), and ibid: 19, 855 (1948), and ibid: 20, 1141 (1949).

[38] International Tables for X-ray Crystallography III, C.A. McGillavary, G.O. Rieck, K. Lonsdale, edts., Kynock Press, Birmingham, UK 1962, Sect. 3.2.

[39] P. A. Lee, G. Beni, Phys. Rev. B15, 286 (1977).
[40] P. M. Colman, H. C. Freeman, J. M. Guss, M. Murata, V. A. Norris, J. A. M. Ramshaw, and M. P. Venkatappa, Nature London 272, 319 (1978).

[41] S. P. Cramer, J. H. Hodgson, and L. P. Hager, J. Amer. Ceram. Soc. 100, 7282 (1978).

[42] S.P. Cramer, W. O. Gillum, K. O. Hodgson, L.E. Mortenson, E. I. Stiefel, J. F. Chisnel, W. J. Brill, and V. K. Shah, J. Amer. Ceram. Soc. 100, 3814 (1978).

[43] G. Martens, P. Rabe, and S. Schartner, Phys. Rev. B17, 1481 (1978).

[44] P. Rabe, Japan J. Appl. Phys. 17, 22 (1978).

[45] L. Incoccia, S. Mobilio, M. G. Proietti, P. Fiorini, C. Giovannella, and F. Evangelisti, Phys. Rev. 31, 1028 (1985).

[46] G. Bunker and E. A. Stern Phys. Rev. Lett. 52, 1990 (1984), and ibid: 54, 2726 (1985).

[47] D. D. Vvedensky and J. B. Pendry, Phys. Rev. Lett. 54, 2725 (1985).

[48] C.E. Bouldin, G. B. Unker, D. A. McKeown, R. A Forman, and J.J. Ritter, Phys. Rev. B38, 10816 (1988).

[49] J. E. Müller and W. L. Schaich, Phys. Rev. B27, 6489 (1983).

[50] W. L. Schaich, Phys. Rev. B29, 6513 (1984).

[51] J. J. Rehr, R. C. Albers, C. R. Natoli, and E. A. Stern, Phys. Rev. B34, 4350 (1986).

[52] P. H. Citrin, P. Eisenberger, and B. M. Kincaid, Phys. Lett. 36, 1346 (1976).

[53] B. Lengeler, J. de Physique, Colloque C8, 75 (1986).

[54] G.S. Brown, P. Eisenberger, and P. Schmidt, Solid State Comm. 24, 201 (1977).

[55] P. Rabe, G. Martens, N. Schwentner, and A. Werner, Phys. Rev. Lett. 39, 1411 (1977).

[56] G. Dalba, P. Fornasini, F. Rocca, E. Bernieri, E. Burattini, and S. Mobilio, J. Non-Cryst. Solids 91, 153 (1987).

[57] S. J. Gurman, J. Mater. Sci. 17, 1541 (1982).

[58] S. Rundqvist, Acta Chem. Scand. 14, 1961 (1960).

[59] B. Aronsson, Acta Chem. Scand. 9, 137 (1955).

[60] A. G. McKale, B. M. Veal, A. P. Paulikas, S. K. Chan, and G.S. Knapp, J. Amer. Chem. Soc. 110, 3763 (1988).

[61] E. A. Stern, B. A. Bunker, and S. M. Heald, Phys. Rev B21, 1346 (1980)

[62] P. A. Lee, B. K. Teo, and A. L. Simons, J. Amer. Chem. Soc. 99, 3856 (1977).

[63] D. E. Sayers, E. A. Stern, and F. W. Lytle, Phys. Rev. Lett. 35, 584 (1975).

[64] S. H. Hunter, A. Bienenstock, and T. M. Hayes, in: The Structure of Non-Crystalline Materials, P. H. Gaskell, edt., Taylor and Francis Ltd. London 1977, p 73.

[65] P. Rabe, G. Tolkiehn, and A. Werner, J. Phys. C12, L545 (1979).

[66] G. Bunker, Nucl. Instrum. Methods 207, 437 (1983).

[67] E. A. Stern, P. Livins, and Z. Zhang, Phys. Rev. B43, 8850 (1991). 